\title{
Novel therapeutic effects of sesamin on diabetes-induced cardiac dysfunction
}

\author{
TRAN DUONG THUY ${ }^{1 *}$, NAM NHUT PHAN ${ }^{2 *}$, CHIH-YANG WANG ${ }^{3-5}$, HAN-GANG YU $^{6}$, \\ SHU-YIN WANG ${ }^{1}$, PUNG-LING HUANG $^{1}$, YI-YIN DO ${ }^{7}$ and YEN-CHANG LIN ${ }^{1}$
}

\author{
${ }^{1}$ Graduate Institute of Biotechnology, Chinese Culture University, YangMingShan, Taipei 11114, Taiwan, R.O.C.; \\ ${ }^{2}$ Faculty of Applied Sciences, Ton Duc Thang University, Ho Chi Minh City 70000, Vietnam; ${ }^{3}$ Department of Anatomy, \\ University of California San Francisco, San Francisco, CA 94143, USA; ${ }^{4}$ Department of Biochemistry and Molecular Biology; \\ ${ }^{5}$ Institute of Basic Medical Sciences, College of Medicine, National Cheng Kung University, Tainan 701, Taiwan, R.O.C.; \\ ${ }^{6}$ Department of Physiology and Pharmacology, West Virginia University, Morgantown, WV 26506, USA; \\ ${ }^{7}$ Department of Horticulture and Landscape Architecture, National Taiwan University, Taipei 10617, Taiwan, R.O.C.
}

Received July 8, 2016; Accepted October 27, 2016

DOI: $10.3892 / \mathrm{mmr} .2017 .6420$

\begin{abstract}
Diabetes is a risk factor that increases the occurrence and severity of cardiovascular events. Cardiovascular complications are the leading cause of mortality of $75 \%$ of patients with diabetes $>40$ years old. Sesamin, the bioactive compound extracted from Sesamum indicum, is a natural compound that has diverse beneficial effects on hypoglycemia and reducing cholesterol. The aim of this study is to investigate sesamin effects to diabetes-inducing cardiac hypertrophy. In the present study bioinformatics analysis demonstrated cardiac hypertrophy signaling may be the most important pathway for upregulating genes in sesamin-treated groups. To verify the bioinformatics prediction, sesamin was used as the main bioactive compound to attenuate the impact of diabetes induced by streptozotocin (STZ) on cardiac function in a rat model. The results revealed that oral administration of sesamin for 4 weeks (100 and $200 \mathrm{mg} / \mathrm{kg}$ body weight) marginally improved blood glucose levels, body weight and significantly ameliorated the effects on heart rate and blood pressure in rats with type 1 diabetes relative to control rats. The QT interval of sesamin was also reduced relative to the control group. The findings indicated that sesamin has potential cardioprotective effects in the STZ-induced diabetes model. This suggested that this can be used as a novel treatment for patients with diabetes with cardiac dysfunction complication.
\end{abstract}

Correspondence to: Professor Yen-Chang Lin, Graduate Institute of Biotechnology, Chinese Culture University, 55 HwaKang Road, YangMingShan, Taipei 11114, Taiwan, R.O.C.

E-mail: lycnthu@gmail.com

${ }^{*}$ Contributed equally

Key words: Sesamin, cardiac dysfunction, diabetes

\section{Introduction}

Diabetes mellitus (DM) is a metabolic disease in patients with high blood glucose over prolonged period (1). It has been well documented as an urgent worldwide health issue present in a wide age range, from children to adults (2). Diabetes leads to numerous complications associated with the heart, including ischemic heart disease, peripheral vascular disease, and cerebrovascular disease (3). Type $1 \mathrm{DM}$ is caused by the autoimmune destruction of the pancreatic $\beta$-cells resulting in reduced insulin production (4). Chronically, microvascular and macrovascular complications are the major reasons for increasing morbidity and mortality in patients with diabetes. In developed countries, cardiovascular disease is the main complication causing death resulted from diabetes $(5,6)$.

Patients with type 1 DM face a two to four times higher mortality rate than non-diabetic people (7-10). The main risk factor raising the mortality and morbidity of type $1 \mathrm{DM}$ is cardiovascular complications. Their impact has become clearer and more pernicious to patients with DM $(7,10)$. Coronary atherosclerosis appears in the majority of the diabetes population, with the mortality of up to $20 \%$ diabetic subjects caused by myocardial infarction (9). Normoglycemia is the key to attenuate the microvascular and neurological complications of type 1 diabetes. There are many methods of maintaining normal blood sugar levels, including treatment with insulin focus to a therapy area by external pumps, blood glucose monitoring and physical exercise. However, there is an increasing interest in using natural foods as herbal treatments, including as diabetes therapeutics (11-14). The pharmacological properties of herbal remedies, including the amelioration of insulin sensitivity, the promotion of insulin secretion, the increase of glucose uptake by adipose and muscle tissues, the inhibition of sugar absorption in intestines and the generation of sugar by hepatocytes, have been demonstrated in several studies $(15,16)$. Herbal and traditional Chinese medicine have also exhibited a strong impact on treatment of cardiovascular complications in diabetes (17). Sesamum indicum has been 
used as a nutrient-rich food with medicinal functions for a many years; it is also known as a precious herb. Sesamin is the bioactive compound that is extracted in high amounts from Sesamum indicum. Sesamin has been used in pharmaceuticals to perform various valuable functions (18). Numerous studies have demonstrated that sesamin provides considerable support to human health by improving fat metabolism, antioxidant activity, hypolipidemic activity, reducing cholesterol, preventing inflammation and enhancing the potency of vitamin E (18-21). However, whether sesamin can regulate diabetes or the cardiac disease network is remains largely unknown.

High-throughput microarray technology is the most powerful tool to analyze gene expression on a global scale, offering measurement of the expression of a huge number of genes. With the rapid expansion of microarray datasets, high-throughput microarray technology has become more popular and more accessible from the Gene Expression Omnibus (GEO) (22), under the management of National Center for Biotechnology Information. Expression data from the GEO (series accession no. GSE42693) was selected (23) for analyzing the potential signal transduction pathways in sesamin-treated rats compared with a group treated with a controlled diet. Previous studies have also demonstrated that sesamin can regulate fatty acid oxidation in rats, while other studies have reported that sesamin can recover left ventricular remodeling in spontaneously hypertensive rats (24). However, the association between diabetes-induced cardiac hypertrophy and sesamin is remains largely unknown.

Sesamin has been revealed to reduce the effects of oxidative stress and nitric oxide synthesis on $\beta$-cell destruction (25). However, to the best of our knowledge, there has been no previous research on the effect of sesamin on cardiac dysfunction in type 1 diabetes. Thus, the current study was a comparative analysis of how sesamin affected diabetesinduced cardiac dysfunction. Based on the bioinformatics analysis within the present study, the microarray network data demonstrated that the cardiac hypertrophy pathway was an important pathway in the sesamin-treated group. Therefore, the present study aimed to investigate the cardioprotective effect of sesamin in rats with type 1 diabetes induced by streptozotocin (STZ).

\section{Materials and methods}

Bioinformatics analysis. Previous research reported that the decline in responsiveness of diabetes-associated heart disease induced by STZ is caused via $\beta$-adrenoreceptor (AR) agonists as a consequent of decrease of $\beta 1-A R$ and the increase in $\beta 3-A R$ expression (26). A report also demonstrated that the maximal left ventricular developed pressure (LVDP) is increased by the effect of $\beta 3$-AR agonists in STZ-induced diabetic rats (24). However, the details of the association between sesamin and diabetic-induced cardiac hypertrophy remain. Thus, the GEO database (http://www.ncbi.nlm.nih.gov/geo/; series accession no. GSE42693) was used to analyze the potential pathways that are stimulated in sesamin-treated rats (23).

From the GSE42693 dataset, male Sprague Dawley (SD) rats with type $1 \mathrm{DM}$ were treated with $0.2 \%$ sesamin in their diet, whereas the control rats were provided with diet free of sesamin for a period of 15 days. RNA extracted from the rats was subjected to microarray analysis. A robust multichip average algorithm was applied to analyze the raw data correction and normalization, and its signal was calculated for gene-level probe set summaries with the Affymetrix Expression Console Version 1.3.1.187 (http://www.affymetrix.com/). Then the fold change and expression level between the sesamin-treated group and the control group were also calculated. MetaCore (GeneGo, Inc., St. Joseph, MI, USA) was employed to investigate the signal transduction pathways regulating the sesamin-treated group by analyzing gene ontology molecular functions and GeneGo pathways. MetaCore received the data input and created biological links with associated processes. In order to compare the average expression level of rats treated with sesamin $(n=5)$ and the control $(n=5)$, the upper quartile of highest expressed genes (7,774 genes) with significant expression differences was uploaded to the MetaCore software. From the analysis, the cardiac hypertrophy pathway was considered to be a crucial pathway in the sesamin-treated group. $\mathrm{P}<0.05$ was considered to indicate a statistically significant difference.

Sesamin preparation and reagents. Pure sesamin compound was purchased from ChemBio Co. (Taipei, Taiwan), a glucose kit was purchased from Randox Laboratories Ltd. (County Antrim, UK) and STZ was purchased from Sigma-Aldrich (Merck Millipore, Darmstadt, Germany).

STZ-induced type I diabetes rat model. A total of 30 male, pathogen and disease-free Sprague-Dawley rats (4-week-old; weight, 100-120 g) from the National Laboratory Animal Center (Taipei, Taiwan), were used in the current study. The rats were raised under standard experimental conditions with a $12 \mathrm{~h}$ light $/ 12 \mathrm{~h}$ dark cycle at $23-25^{\circ} \mathrm{C}$. Animals were fed ad libitum with free access to water. All animal experiments were approved by the Institutional Animal Care and Use Committee or Panel (IACUC/IACUP) of Chinese Culture University (Taipei, Taiwan). The diabetes rat model was created in 10 rats by STZ injection following a standard protocol of the Animal Models of Diabetic Complications Consortium (27). STZ was diluted in sodium citrate buffer solution $(\mathrm{pH} 4.5)$. The rats were fasted for $4 \mathrm{~h}$ before the STZ injection and then were anesthetized using $2 \%$ isoflurane mixed with $21 / \mathrm{min}$ compressed oxygen (KYS Technology, Taipei, Taiwan). An appropriate amount of the STZ solution $(50 \mathrm{mg} / \mathrm{kg})$ was injected subcutaneously in the abdomen area, repeating one injection each day for five consecutive days. Each rat was supplied with $10 \%$ sucrose water following each injection, was allowed to awaken and was placed back in the cage. The rats were tested for sufficient levels of hyperglycemia and only rats with blood glucose level $>200 \mathrm{mg} / \mathrm{dl}$ (5 rats) were selected for further experimentation.

Sesamin treatment in STZ-induced diabetic rats. A total of 5 groups of experiments with 5 rats of each were used in this study (control, model diabetes, and sesamin treatment (3 groups). Sesamin (dissolved in olive oil) oral administration was performed after the STZ-treated rats were randomly divided into three groups that received different sesamin concentrations as follows: Low concentration $(50 \mathrm{mg} / \mathrm{kg}$ body weight), medium concentration (100 $\mathrm{mg} / \mathrm{kg}$ body weight), and high concentration ( $200 \mathrm{mg} / \mathrm{kg}$ body weight). The experimental 
process was performed for 4 weeks (oral administration of sesamin to rats was performed once per day), and after each 7-day period of treatment with sesamin, blood samples were collected in order to determine the blood glucose levels. Body weight, heart rate (HR) and blood pressure (BP) were also measured, and echocardiogram was performed.

Blood glucose measurement. The rats were fasted for $4 \mathrm{~h}$ before the blood sample collections on day $0,7,14,21$ and 28 of the sesamin administration process. Serum was separated for the measurement of the blood glucose, using commercial kits according to the manufacturer's protocols (Randox Laboratories, Ltd., County Antrim, UK).

Blood pressure and electrocardiogram measurements. BP was measured by inserting two catheters into the femoral artery. For BP, the custom catheter (polyurethane; 1 French size) was inserted to the femoral artery. The waveforms delivering information about LVDP were visualized on a computer monitor.

The electrocardiogram was measured by using three lead vectors, one connected to the chest, one connected to the armpit, and the third connected to the femoral part of the rat. All results were recorded and analyzed by the LabChart 7 software (ADInstruments, Ltd., Dunedin, New Zealand).

Histopathological assay. Rats were scarified by anesthetizing with $2 \%$ isoflurane (KYS Technology, Taipei, Taiwan) mixed with $21 / \mathrm{min}$ of compressed oxygen and followed by decapitalization. The hearts were collected and stored in $10 \%$ formalin at $4^{\circ} \mathrm{C}$ for subsequent paraffin embedding. Sections $(5 \mu \mathrm{m})$ of each tissue were stained with hematoxylin and eosin $(\mathrm{H} \& \mathrm{E})$ staining and were observed for histopathological alterations under a light microscope.

Data collection and analysis. Heart rate measurements, electrocardiography performance and blood pressure recording was performed using cardiovascular continuous monitoring software (PowerLab/4SP; ADInstruments, Ltd.). Values for hemodynamic measures were derived by a $1 \mathrm{KHz}$ sampling rate.

Statistical analysis. All results are presented as the mean \pm standard deviation. Statistical analysis was evaluated by one-way analysis of variance followed by the StudentNewman-Keuls test with SPSS software (version 16.0; SPSS, Inc., Chicago, IL, USA). $\mathrm{P}<0.05$ was considered to indicate a statistically significant difference.

\section{Results}

Cardiac hypertrophy pathways are modulated by sesamin treatment. MetaCore GeneGo software was used to identify genes with different expression levels in sesamin-treated and control samples. MetaCore software employs an exclusive, manually selected and organized database comprising of protein-protein, protein-DNA, protein compound interactions, and their metabolic and signaling pathways. There were 7,774 genes with significant expression differences between the sesamin-treated $(n=5)$ and the control diet-treated rats $(n=5)$.
MetaCore pathway analysis of these two groups indicated that most significant signaling pathway for upregulated genes in sesamin-treated rats was cardiac hypertrophy signaling $(\mathrm{P}=1.148 \mathrm{E}-10$ and $\mathrm{FDR}=9.945 \mathrm{E}-8)$.

Cardiac hypertrophy is a compensatory mechanism of the heart to maintain stable cardiac output throughout different medical states. The outcome of this chronic process results in pathological cardiac growth and is correlated with increased morbidity and mortality. Myocyte enhancer factor 2 (MEF2) family proteins (MEF2A, MEF2C, MEF2D) are involved as intermediate signal responses of the cardiac transcriptional program $(26,28,29)$. Numerous genes serve roles in the cardiac hypertrophy process, including $\alpha$-myosin $(\alpha-\mathrm{MHC})$ and $\beta$-MHC, troponin I cardiac and troponin $\mathrm{T}$ cardiac, via a system of transcription factors and co-factors (GATA binding protein 4 , NK2 homeobox 5, calmodulin binding transcription activator 2, MEF2, heart and neural crest derivatives expressed 1 (HAND1), HAND2, CREB binding protein and E1A binding protein p300) incorporating with nuclear factor of activated T-cells (NF-AT) signaling (30-32). The bioinformatics results suggest that sesamin may participate in the cardiac hypertrophy pathway via NF-AT signaling (Fig. 1). However, currently, the detailed mechanism of how sesamin affects cardiac hypertrophy is still obscured and demands more investigation in animal model.

Histology of the cardiac tissue. Results from H\&E staining demonstrated that the nuclei in the control group were oval, single and observed at the center of the cardiomyocytes (Fig. 2). However, deformation in the shapes and sizes of nuclei was observed in the diabetes group. In addition, the cardiac myofiber pattern in this group spread irregularly relative to the control group. An increase in protein degradation may lead to this observation. Compared with the diabetic group, serious histological changes in the sesamin oral administration group were reduced. Among the treatment groups, the mediumdose group improved features compared with the diabetes group. Massive fibroblast infiltration in the left ventricle was observed in the diabetes group. Treatment with different doses of sesamin induced different changes in the diabetes rats. In the low-dose group, the cardiomyocytes degenerated, and segmentation was observed after 4 weeks of sesamin administration. Even mature fibrocyte infiltration was observed in this group. In the high-dose group, the staining indicated degenerated, vacuolated and dissociated myocardiac fibers in the focal region of the left ventricle. By contrast, the heart tissue improved greatly after 4 weeks of oral treatment with the medium sesamin dose.

Effect of sesamin on blood glucose and body weight. Following STZ injection, SD rats exhibited high glucose blood level (>200 mg/dl). At day 28th of the treatment, The STZ-treated rats exhibited a reduction in body weight $(160.2 \pm 6.49 \mathrm{~g}, \mathrm{n}=5)$ and an increase in blood glucose $(220.47 \pm 6.19 \mathrm{mg} / \mathrm{dl}, \mathrm{n}=5)$ compared with the age-matched controls, with $200.4 \pm 6.34 \mathrm{~g}$ and $107.81 \pm 6.69 \mathrm{mg} / \mathrm{dl}$, respectively (Fig. 3A). However, following oral sesamin administration, there was significant glycemic amelioration compared with the diabetes group by $17 \%$ in the low dose group, by $30 \%$ in the medium dose group and by $26 \%$ in the high dose group. Body weight was 

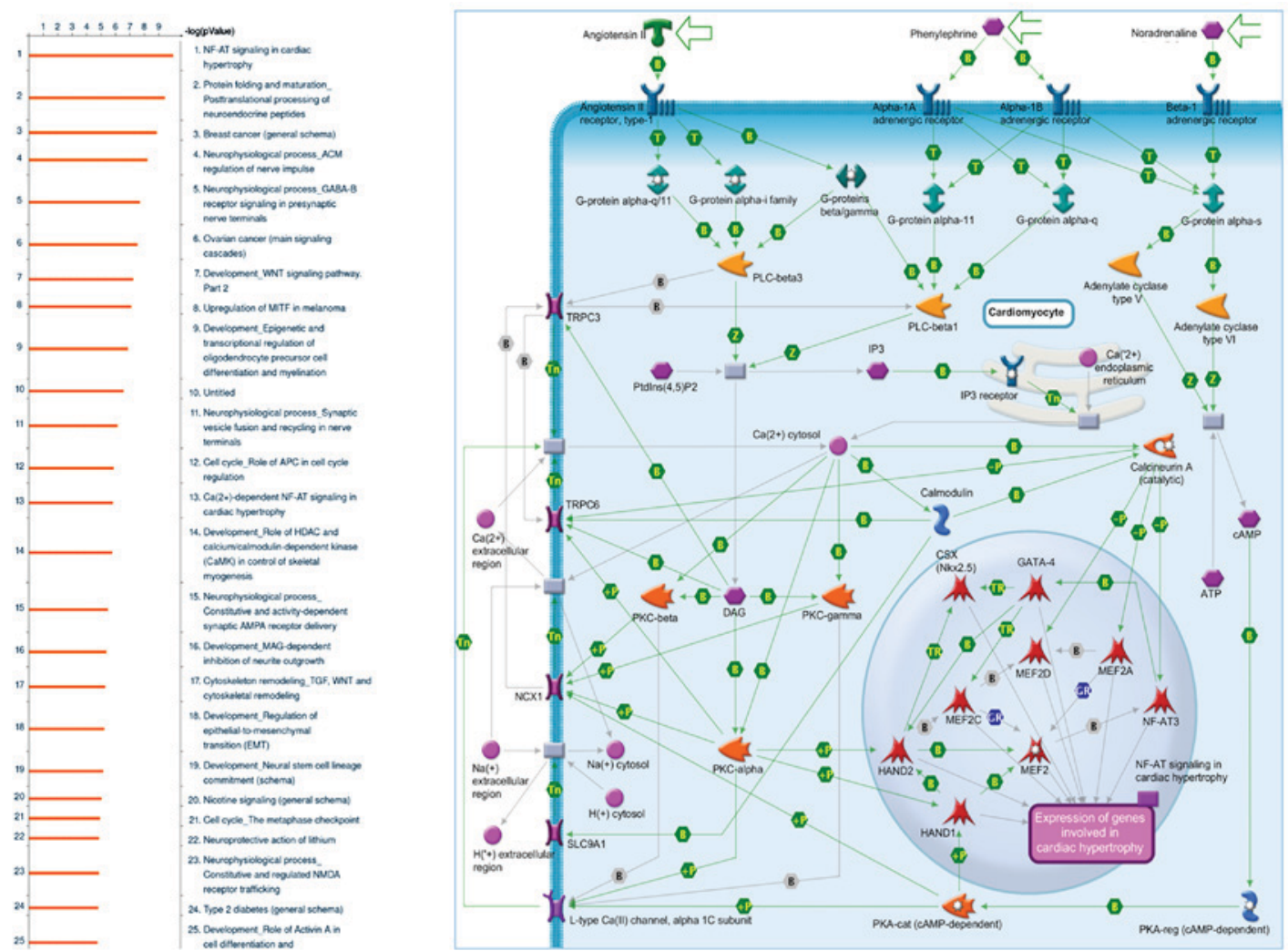

Figure 1. Meta Core pathway analysis indicated that cardiac hypertrophy signaling was significantly associated with sesamin-treated samples $(\mathrm{P}=1.148 \mathrm{E}-10)$. Green arrows indicate activation.

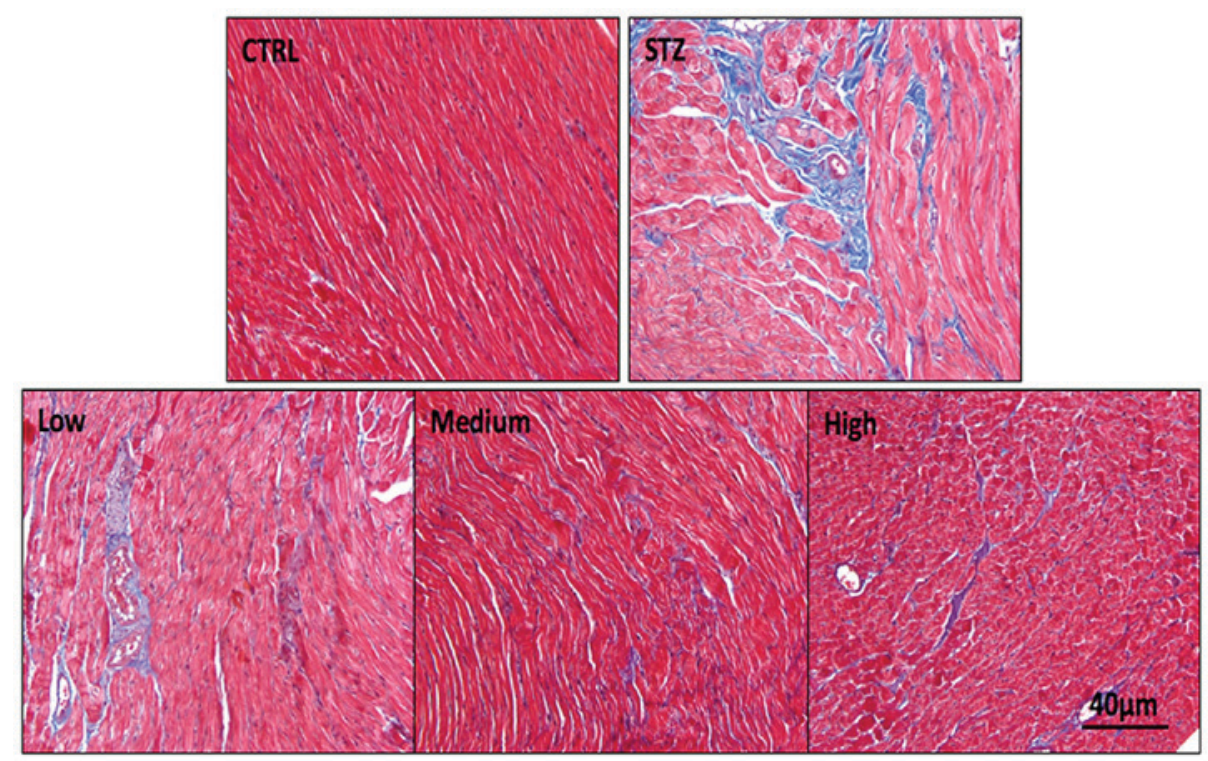

Figure 2. Histological examination of cardiac tissue from the control group, diabetes group (STZ), low-dose sesamin oral administration group, medium-dose sesamin oral administration group and high-dose sesamin oral administration group. CRTL, control; STZ, streptozotocin.

increased compared with the diabetes group by $17 \%$ in the low dose group, $18 \%$ in the medium dose group, and $20 \%$ in the high dose group at 4 weeks (Fig. 3B). The body weight was increased in the sesamin-treated groups compared with the diabetes group, however, for the groups administered medium and high doses of sesamin, blood glucose levels were reduced further than in the low dose group.
Effect of sesamin on blood pressure. After STZ-induction, at day 28th of the treatment, the systolic and diastolic blood pressure in the diabetic subjects $(85.46 \pm 0.92$ and $55.59 \pm 1.06 \mathrm{mmHg}$ ) was markedly reduced compared with the control subjects $(123.12 \pm 1.54$ and $81.90 \pm 1.09 \mathrm{mmHg}, \mathrm{n}=5)$ (Fig. 3C). Following sesamin consumption, blood pressure was significantly increased in STZ-induced rats, particularly 
A

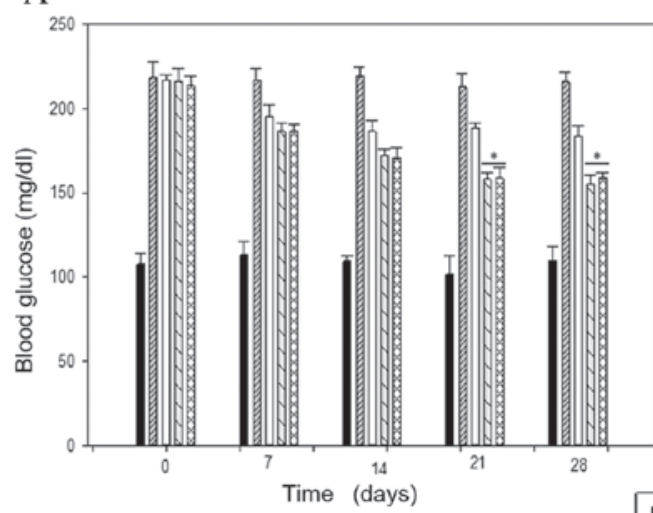

B

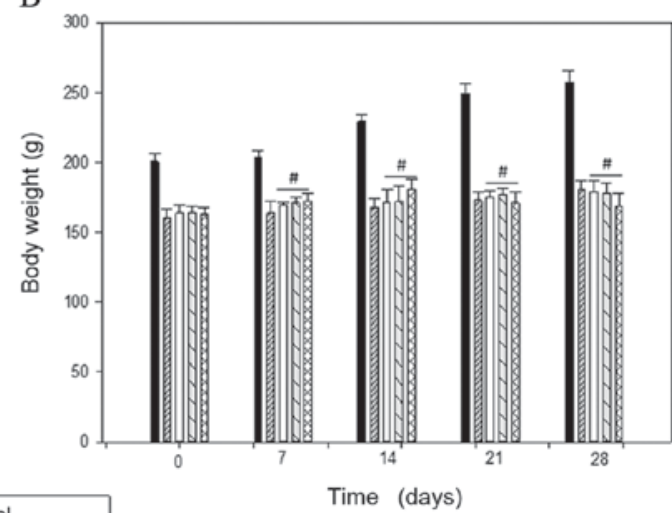

\section{Control
Lowel diabetes
Low dose Low dose \&os High dose}
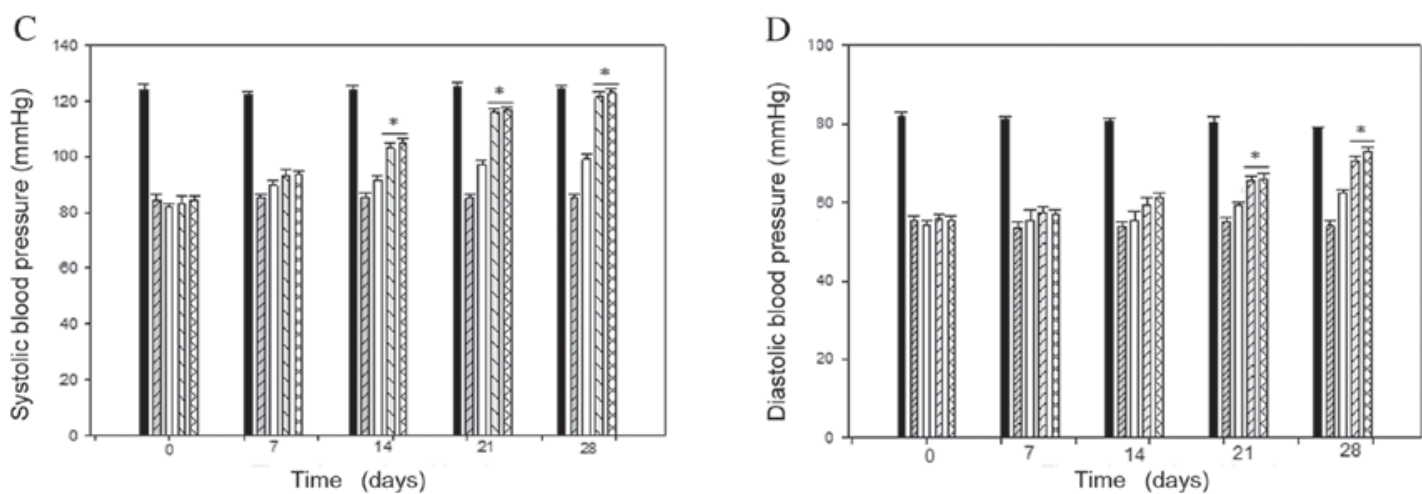

Figure 3. Effect of sesamin on (A) fasting plasma glucose, (B) body weight, (C) systolic bold pressure and (D) diastolic blood pressure of streptozotocin-treated Sprague-Dawley rats. Data are presented as the mean \pm standard deviation $\left(\mathrm{n}=5 ;{ }^{*} \mathrm{P}<0.05\right.$ vs. low-dose group; ${ }^{*} \mathrm{P}<0.05$ vs. model diabetes).

in the groups administered medium and high doses of sesamin, with the low-dose group from $82.28 \pm 0.98 / 54.34 \pm 0.99 \mathrm{mmHg}$ to $99.15 \pm 1.59 / 62.49 \pm 0.82 \mathrm{mmHg}$, the medium-dose group from $83.74 \pm 0.87$ to $121.47 \pm 1.09 / 55.96 \pm 1.67 / 70.67 \pm 1.05$, and the high-dose group from $84.53 \pm 1.19 / 55.48 \pm 1.16 \mathrm{mmHg}$ to 123.14 $\pm 1.21 / 72.99 \pm 1.14 \mathrm{mmHg}$ (Fig. 3D).

Effect of sesamin on heart rate. The heart rate in rats injected with STZ declined by $\sim 1.33$-fold compared with the healthy subjects $(333.6 \pm 10.21$ vs. $249.4 \pm 12.17 \mathrm{BPM})$. The heart rate was significantly increased following treatment with sesamin compared with the diabetes group, particularly in the highdose sesamin group at day 28th of the treatment (255 \pm 9.61 vs. $308 \pm 5.65$ BPM; Fig. 4A).

The effect of sesamin on QT interval. The uncorrected QT interval was prolonged in STZ-treated rats compared with the control group at day 28 th of the treatment $(73.29 \pm 3.31$ vs. 54.71 $\pm 2.18 \mathrm{msec})$. Following sesamin treatment, the QT interval dramatically recovered by $7.4,11.5$ and $15.2 \%$ at day 28 th of the treatment with the low-dose, medium-dose and high-dose group, respectively, compared with the STZ-treated rats (Fig. 4B). Fridericia's formula was used to correct the QT interval by adjusting to an R-R interval. Corrected QT intervals were $82.67 \pm 3.99$ and $63.28 \pm 1.77 \mathrm{msec}$ in STZ-treated rats and control rats at day 28 th of the treatment, respectively. There was a corrected QT interval of 8,12 and $14 \%$ in the low-dose, medium-dose and high-dose groups at day 28th of the treatment, respectively (Fig. 4C).

\section{Discussion}

Cardiac fibrosis and pressure dysfunction in patients with diabetes were reported in a previous study (33). Diabetic cardiomyopathy in rats with type 2 diabetes causes an increase in heart weight and in the nuclei size in cardiomyocytes. However, in rats with type 1 diabetes, studies by Cosyns et al (34) and Thent et al (35) demonstrated the same result, where the disarray in cardiac myofibers and changes in nuclei size led to a decrease in the cardiomyocytes, which caused cardiac atrophy $(34,35)$. Cardiac atrophy may be due to an insulin deficiency, leading to a decline in protein synthesis and serious protein degradation, which affects the function of the mitochondria and leads to the damage of myocardial myofibers (36). Other causes that can lead to atrophy of the heart include an increase in hyperosmolarity in hyperglycemic conditions, an increase of collagen bundles, and a rise in the left ventricle contraction; all of these may lead to the changing of nuclei sizes and the shapes of cardiomyocytes (37). Sesame contains large amounts of antioxidants such as flavonoids and vitamin C and E. Sesamin extracted from Sesamum indicum has anti-inflammatory effects, supports vitamin $\mathrm{E}$ absorption, and reduces $\beta$ cell-destroying factors, therefore, increasing the amount of insulin, ameliorating hyperglycemia, and increasing 
A
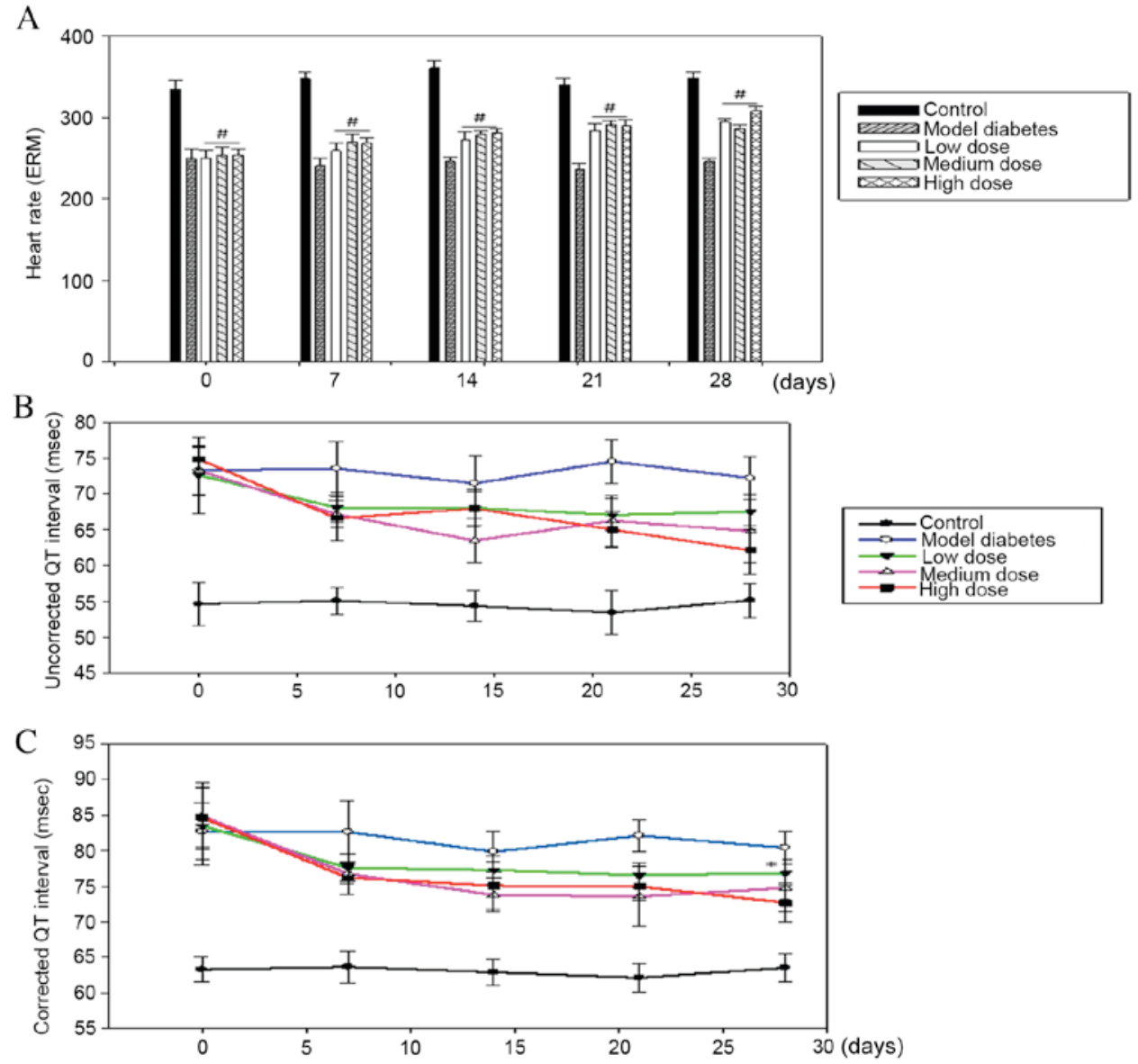

Figure 4. Effect of sesamin on (A) heart rate, (B) QT interval and (C) corrected QTcF (msec) interval (using the Fridericia formula) following oral sesamin administration. ${ }^{*} \mathrm{P}<0.05$ vs. controls; ${ }^{~} \mathrm{P}<0.05$ vs. model diabetes.

protein synthesis (38). Through the anti-inflammatory effects of sesamin, the myocardial injury also somewhat improved. It reduced the contraction of the left ventricle, decreasing the size and changing the shape of the nuclei of myocardial cells, thus, improving the disorder in the myofiber arrangement (39). In the current study, the results of $\mathrm{H} \& \mathrm{E}$ staining demonstrated that structural cardiomyocytes in rats with diabetes induced by STZ were damaged. The order links among the myofibers were broken, and the nuclei of cardiac muscle cells were deformed and fragmented in the diabetic group. However, in the treatment groups, the disordered associations were markedly minimized, particularly in the medium-dose group. Findings of myocardial injury were also reduced. But these injuries were still visible in the low and high sesamin concentration groups.

The combined consumption of sesame oil and glibenclamide reduces blood glucose levels significantly (36\% with treatment compared with no treatment), and it also decreases total plasma cholesterol; this significantly improves the condition of patients with diabetic disease (40). An STZ-induced diabetic heart has been revealed exhibit decreased responsiveness via $\beta$-AR agonists, in which reduced $\beta 1-A R$ and increased $\beta 3-A R$ expression are important factors (26). In addition, a $\beta 3-A R$ agonist also raised the maximal LVDP responses in rats with STZ-induced diabetes (41). The data of the current study demonstrated that following STZ injection, the body weight and blood glucose level of rats declined rapidly and markedly, which has also been described in a previous study (42). This is explained by the destruction of $\beta$ cells induced by STZ (43). In a previous study, significant improvements in the blood sugar levels and body weight of rats were observed (44). However, there was no marked change in the body weight of diabetes rats treated with sesamin relative to model diabetes rat in the current study.

Following oral administration of sesamin, the blood glucose level of rats was significantly reduced compared with STZ-induced rats, particularly at the medium and high sesamin concentrations. This demonstrates that sesamin has a marked hypoglycemic effect; the previous studies also reported a similar outcome $(45,46)$. However, the results in Fig. 1 indicate that using the correct sesamin concentration is critical because the reduction in blood glucose levels is was not significant at the low sesamin concentration.

The data of the present study demonstrated that STZ-induced diabetes results in a decrease in blood pressure and that sesamin treatment can restore blood pressure in diabetic rats. Following oral sesamin administration, the blood pressure was restored significantly. This is reflected in the recovery of the diastolic and systolic blood pressure. Previous studies reported a significant attenuation of vascular dysfunction following treatment with sesamin (44). Possibly the recovery of the blood vessels in mice with sesamin treatment led to the recovery of the blood pressure. Endothelial cells are involved in the restoration of blood vessel walls. Due 
to these cells, the improvement of the blood vessels via the sesamin treatment was considerably increased (44).

Another study demonstrated that sesamin had an effect on the protection of $\beta$ cells following STZ-induction. The administration of sesamin reversed the STZ-induced cells viability reduction. Increasing the oxidative stressors and nitride oxide synthesis by sesamin led to reduced $\beta$ cells destruction (41). In rats with STZ-induced diabetes, the heart rate was decreased in the present study, which is consistent with what has been revealed in previous studies $(47,48)$. Hyperglycemia appears to be the predominant cause of this clinical abnormality (slow heart rate) (48). Also, the effect of STZ on repolarization and the action potential may have led to heart rate reduction (44). An increased heart rate in patients with type 1 diabetes is closely associated with cardiac autonomic neuropathy, thus, leading to many abnormal problems, including high blood pressure and poor blood supply (47). Due to the effect of sesamin treatment, the $\beta$ cells were significantly restored, causing reduced glycemia and leading to a recovered heart rate in a previous report (38). Improving blood pressure is involved in the effect of sesamin that led to the heart rate recovery in STZ-treated animals (44).

QT interval measurement can predict sudden cardiac death in certain diseases, including hypertension (49), chronic heart failure $(50,51)$ and peripheral vascular disease (52). QT interval is a good factor to predict cardiac malfunction and state in patients with diabetes $(53,54)$. A previous study reported a significant reduction of the QT interval in subjects with type 1 diabetes; however, when adjusted by RR intervals, the difference was abolished (55). The previous study also reported a prolonged QT interval (by $235 \%$ ) (56). The correction of the QT interval was computed by Fridericia's formulation:

$$
\mathrm{QTcF}=\frac{\mathrm{QT}}{\sqrt[3]{\mathrm{RR}}}
$$

The formulation used the cube root of RR (53). This difference was maintained in QTcF (prolonged 30\%). This may explain by the decline in heart rate affected by high blood sugar and high blood pressure. This difference was maintained in the corrected QT interval in the current study.

Following oral administration of sesamin, the QT interval and QTcF were markedly improved, by 15.2 and $14 \%$ at day 28 th of treatment, respectively, in the high-dose group. This suggests that sesamin has a positive effect in restoring the QT interval in an STZ-induced type 1 diabetes rat. The restoration of blood pressure and $\beta$ cells $(38,44)$ reduced the blood glucose levels, leading to a reduction in the heart rate and substantial improvement of the QT interval. The beneficial effects of sesamin have been demonstrated through various previous studies (57-62). The data of the current revealed that sesamin has a positive role in improving cardiovascular dysfunction in rats with STZ-induced type 1 diabetes.

In conclusion, cardiovascular dysfunction has caused more mortality among patients than any other complications of type 1 diabetes. The oral administration of sesamin for 4 weeks significantly ameliorated the changes to heart rate, blood pressure and QT interval in STZ-induced type 1 diabetes. This indicates that sesamin has a potential therapeutic effect in reducing cardiovascular complications in diabetic subjects.

\section{Acknowledgments}

Computational analyses and data mining were performed using the system provided by the Bioinformatics Core at the National Cheng Kung University (Tainan, Taiwan), supported by the National Science Council Taiwan. The study was supported by the National Science Council Taiwan of the Executive Yuan (grant nos. NSC 101-2320-B-034-001 and 104-2320-B$034-003)$ and the Ministry of Science and Technology, Taiwan (grant nos. 104-2917-I-006-002, MOST103-2325-B006-012 and 104-2917-I-006-002).

\section{References}

1. Alberti KG and Zimmet PZ: Definition, diagnosis and classification of diabetes mellitus and its complications. Part 1: Diagnosis and classification of diabetes mellitus provisional report of a WHO consultation. Diabet Med 15: 539-553, 1998.

2. Organization WH: Diabetes programme: Facts and figures. http://www/who. int/diabetes/facts/world_figures/en/. Accessed December 17: 1990-1999, 2007.

3. UK prospective diabetes study (UKPDS). VIII. Study design, progress and performance. Diabetologia 34: 877-890, 1991.

4. Atkinson MA and Maclaren NK: The pathogenesis of insulindependent diabetes mellitus. N Engl J Med 331: 1428-1436, 1994.

5. Buowari OY: Diabetes mellitus in developing countries and case series. INTECH Open Access Publisher 2013.

6. Laing SP, Swerdlow AJ, Slater SD, Burden AC, Morris A, Waugh NR, Gatling W, Bingley PJ and Patterson CC: Mortality from heart disease in a cohort of 23,000 patients with insulintreated diabetes. Diabetologia 46: 760-765, 2003.

7. Dahl-Jørgensen K, Larsen JR and Hanssen KF: Atherosclerosis in childhood and adolescent type 1 diabetes: Early disease, early treatment? Diabetologia 48: 1445-1453, 2005.

8. Duca L, Sippl R and Snell-Bergeon JK: Is the risk and nature of CVD the same in type 1 and type 2 diabetes? Curr Diab Rep 13: 350-361, 2013.

9. Mahgoub MA and Abd-Elfattah AS: Diabetes mellitus and cardiac function. Mol Cell Biochem 180: 59-64, 1998.

10. Marcovecchio ML, Tossavainen PH and Dunger DB: Prevention and treatment of microvascular disease in childhood type 1 diabetes. Br Med Bull 94: 145-164, 2010.

11. Hong YJ, Kim N, Lee K, Hee Sonn C, Eun Lee J, Tae Kim S, Ho Baeg I and Lee KM: Korean red ginseng (Panax ginseng) ameliorates type 1 diabetes and restores immune cell compartments. J Ethnopharmacol 144: 225-233, 2012.

12. Watanabe K, Matsuura K, Gao P, Hottenbacher L, Tokunaga H, Nishimura K, Imazu Y, Reissenweber H and Witt CM: Traditional Japanese Kampo medicine: Clinical research between modernity and traditional medicine-the state of research and methodological suggestions for the future. Evid Based Complement Alternat Med 2011: 513842, 2011.

13. Ikemoto T, Sugimoto K, Takita M, Shimoda M, Noguchi H, Naziruddin B, Levy MF, Shimada M and Matsumoto S: Japanese herbal medicine TJ-48 prevents autoimmune diabetes in NOD mice. Am J Chin Med 39: 743-756, 2011.

14. Li WL, Zheng HC, Bukuru J and De Kimpe N: Natural medicines used in the traditional Chinese medical system for therapy of diabetes mellitus. J Ethnopharmacol 92: 1-21, 2004.

15. Li GQ, Kam A, Wong KH, Zhou X, Omar EA, Alqahtani A, Li KM, Razmovski-Naumovski V and Chan K: Herbal medicines for the management of diabetes. Adv Exp Med Biol 771: 396-413, 2012.

16. Cui TH and Li YY: Treatment of type 2 diabetes mellitus oral Chinese patent medicine literature metrology analysis. Zhongguo Zhong Yao Za Zhi 37: 2649-2652, 2012 (In Chinese).

17. Ceylan-Isik AF, Fliethman RM, Wold LE and Ren J: Herbal and traditional Chinese medicine for the treatment of cardiovascular complications in diabetes mellitus. Curr Diabetes Rev 4: 320-328, 2008.

18. Jeng K and Hou R: Sesamin and sesamolin: Nature's therapeutic lignans. Current Enzyme Inhibition 1: 11-20, 2005.

19. Kiso Y: Antioxidative roles of sesamin, a functional lignan in sesame seed, and it's effect on lipid- and alcohol-metabolism in the liver: A DNA microarray study. Biofactors 21: 191-196, 2004. 
20. Ogawa H, Sasagawa S, Murakami T and Yoshizumi H: Sesame lignans modulate cholesterol metabolism in the stroke-prone spontaneously hypertensive rat. Clin Exp Pharmacol Physiol Suppl 22 (Suppl): S310-S312, 1995.

21. Kong X, Yang JR, Guo LQ, Xiong Y, Wu XQ, Huang K and Zhou Y: Sesamin improves endothelial dysfunction in renovascular hypertensive rats fed with a high-fat, high-sucrose diet. Eur J Pharmacol 620: 84-89, 2009

22. Edgar R, Domrachev M and Lash AE: Gene Expression Omnibus: NCBI gene expression and hybridization array data repository. Nucleic Acids Res 30: 207-210, 2002.

23. Ide T, Lim JS, Odbayar TO and Nakashima Y: Comparative study of sesame lignans (sesamin, episesamin and sesamolin) affecting gene expression profile and fatty acid oxidation in rat liver. J Nutr Sci Vitaminol (Tokyo) 55: 31-43, 2009.

24. Li WX, Kong X, Zhang JX and Yang JR: Long-term intake of sesamin improves left ventricular remodelling in spontaneously hypertensive rats. Food Funct 4: 453-460, 2013.

25. Lei H, Han J, Wang Q, Guo S, Sun H and Zhang X: Effects of sesamin on streptozotocin (STZ)-induced NIT-1 pancreatic $\beta$-cell damage. Int J Mol Sci 13: 16961-16970, 2012.

26. Dinçer UD, Bidasee KR, Güner S, Tay A, Özçelikay AT and Altan VM: The effect of diabetes on expression of beta1-, beta2-, and beta3-adrenoreceptors in rat hearts. Diabetes 50: 455-461, 2001.

27. Kolb H: Mouse models of insulin dependent diabetes: Low-dose streptozocin-induced diabetes and nonobese diabetic (NOD) mice. Diabetes Metab Rev 3: 751-778, 1987.

28. Okatan EN, Tuncay E, Hafez G and Turan B: Profiling of cardiac $\beta$-adrenoceptor subtypes in the cardiac left ventricle of rats with metabolic syndrome: Comparison with streptozotocin-induced diabetic rats. Can J Physiol Pharmacol 93: 517-525, 2015.

29. Kasler HG, Victoria J, Duramad O and Winoto A: ERK5 is a novel type of mitogen-activated protein kinase containing a transcriptional activation domain. Mol Cell Biol 20: 8382-8389, 2000.

30. Xu J, Gong NL, Bodi I, Aronow BJ, Backx PH and Molkentin JD: Myocyte enhancer factors $2 \mathrm{~A}$ and $2 \mathrm{C}$ induce dilated cardiomyopathy in transgenic mice. J Biol Chem 281: 9152-9162, 2006.

31. Morin S, Pozzulo G, Robitaille L, Cross J and Nemer M: MEF2dependent recruitment of the HAND1 transcription factor results in synergistic activation of target promoters. J Biol Chem 280 32272-32278, 2005.

32. Bhavsar PK, Dellow KA, Yacoub MH, Brand NJ and Barton PJ: Identification of cis-acting DNA elements required for expression of the human cardiac troponin I gene promoter. J Mol Cell Cardiol 32: 95-108, 2000

33. Hayat SA, Patel B, Khattar RS and Malik RA: Diabetic cardiomyopathy: Mechanisms, diagnosis and treatment. Clin Sci (Lond) 107: 539-557, 2004

34. Cosyns B, Droogmans S, Weytjens C, Lahoutte T, Van Camp G, Schoors D and Franken PR: Effect of streptozotocin-induced diabetes on left ventricular function in adult rats: An in vivo Pinhole Gated SPECT study. Cardiovasc Diabetol 6: 30, 2007.

35. Thent ZC, Lin TS, Das S and Zakaria Z: Histological changes in the heart and the proximal aorta in experimental diabetic rats fed with Piper sarmentsoum. Afr J Tradit Complement Altern Med 9: 396-404, 2012.

36. Nemoto O, Kawaguchi M, Yaoita H, Miyake K, Maehara K and Maruyama Y: Left ventricular dysfunction and remodeling in streptozotocin-induced diabetic rats. Circ J 70: 327-334, 2006.

37. Malone JI, Schocken DD, Morrison AD and Gilbert-Barness E: Diabetic cardiomyopathy and carnitine deficiency. J Diabetes Complications 13: 86-90, 1999

38. Lei H, Han J, Wang Q, Guo S, Sun H and Zhang X: Effects of sesamin on streptozotocin (STZ)-induced NIT-1 pancreatic beta-cell damage. Int J Mol Sci 13: 16961-16970, 2012.

39. Utsunomiya T, Shimada M, Rikimaru T, Hasegawa $H$, Yamashita Y, Hamatsu T, Yamasaki M, Kaku S, Yamada K and Sugimachi K: Antioxidant and anti-inflammatory effects of a diet supplemented with sesamin on hepatic ischemia-reperfusion injury in rats. Hepatogastroenterology 50: 1609-1613, 2003

40. Sankar D, Ali A, Sambandam G and Rao R: Sesame oil exhibits synergistic effect with anti-diabetic medication in patients with type 2 diabetes mellitus. Clin Nutr 30: 351-358, 2011.

41. Okatan EN, Tuncay E, Hafez G and Turan B: Profiling of cardiac $\beta$-adrenoceptor subtypes in the cardiac left ventricle of rats with metabolic syndrome: Comparison with streptozotocin-induced diabetic rats. Can J Physiol Pharmacol 93: 517-525, 2015.
42. Howarth FC, Jacobson M, Shafiullah M and Adeghate E: Long-term effects of streptozotocin-induced diabetes on the electrocardiogram, physical activity and body temperature in rats. Exp Physiol 90: 827-835, 2005

43. Akbarzadeh A, Norouzian D, Mehrabi MR, Jamshidi SH, Farhangi A, Verdi AA, Mofidian SM and Rad BL: Induction of diabetes by Streptozotocin in rats. Indian J Clin Biochem 22: 60-64, 2007.

44. Baluchnejadmojarad T, Roghani M, Jalali Nadoushan MR, Vaez Mahdavi MR, Kalalian-Moghaddam H, Roghani-Dehkordi F, Dariani S and Raoufi S: The sesame lignan sesamin attenuates vascular dysfunction in streptozotocin diabetic rats: Involvement of nitric oxide and oxidative stress. Eur J Pharmacol 698: 316-321, 2013.

45. Hong L, Yi W, Liangliang C, Juncheng H, Qin W and Xiaoxiang Z: Hypoglycaemic and hypolipidaemic activities of sesamin from sesame meal and its ability to ameliorate insulin resistance in KK-Ay mice. J Sci Food Agric 93: 1833-1838, 2013.

46. Sankar D, Ali A, Sambandam G and Rao R: Sesame oil exhibits synergistic effect with anti-diabetic medication in patients with type 2 diabetes mellitus. Clin Nutr 30: 351-358, 2011.

47. Turker Y, Aslantas Y, Aydin Y, Demirin H, Kutlucan A, Tibilli $\mathrm{H}$, Turker $\mathrm{Y}$ and Ozhan H: Heart rate variability and heart rate recovery in patients with type 1 diabetes mellitus. Acta Cardiol 68: 145-150, 2013

48. Jaiswal M, Urbina EM, Wadwa RP, Talton JW, D'Agostino RB Jr, Hamman RF, Fingerlin TE, Daniels S, Marcovina SM, Dolan LM and Dabelea D: Reduced heart rate variability among youth with type 1 diabetes: The SEARCH CVD study. Diabetes Care 36: 157-162, 2013.

49. Sahu P, Lim PO, Rana BS and Struthers AD: QT dispersion in medicine: Electrophysiological holy grail or fool's gold? QJM 93: 425-431, 2000

50. Barr CS, Naas A, Freeman M, Lang CC and Struthers AD: QT dispersion and sudden unexpected death in chronic heart failure. Lancet 343: 327-329, 1994.

51. Fu GS, Meissner A and Simon R: Repolarization dispersion and sudden cardiac death in patients with impaired left ventricular function. Eur Heart J 18: 281-289, 1997.

52. Darbar D, Luck J, Davidson N, Pringle T, Main G, McNeill G and Struthers AD: Sensitivity and specificity of QTc dispersion for identification of risk of cardiac death in patients with peripheral vascular disease. BMJ 312: 874-879, 1996.

53. Naas AA, Davidson NC, Thompson C, Cummings F, Ogston SA, Jung RT, Newton RW and Struthers AD: QT and QTc dispersion are accurate predictors of cardiac death in newly diagnosed noninsulin dependent diabetes: Cohort study. BMJ 316: 745-746, 1998.

54. Rana BS, Lim PO, Naas AA, Ogston SA, Newton RW, Jung RT, Morris AD and Struthers AD: QT interval abnormalities are often present at diagnosis in diabetes and are better predictors of cardiac death than ankle brachial pressure index and autonomic function tests. Heart 91: 44-50, 2005.

55. Howarth FC, Adeghate E and Jacobson M: Heart rate and QT interval in streptozotocin-induced diabetic rat. J Med Sci 2: 108-118, 2009.

56. Fridericia LS: The duration of systole in an electrocardiogram in normal humans and in patients with heart disease. 1920. Ann Noninvasive Electrocardiol 8: 343-351, 2003.

57. Fukuda Y, Osawa T,Namiki M and Ozaki T: Studies on antioxidative substances in sesame seed. Agric Biol Chem 49: 301-306, 1985.

58. Ying D and Yin G: Extraction of Antioxidant from Sesame Cake and Its Activity Study. Food Science 2: 2007.

59. Bian HL: Effect of sesamin on immune function and proliferating cell nuclear antigen in S180 sarcoma-bearing mice. China J Mod Med 18: 3411-3417, 2008

60. Wei YJ, Bian HL and Zhao CF: Inhibitory effect of sesamin on tumor growth and mechanism in $\mathrm{H}(22)$ hepatoma-bearing mice. Shandong Med J 19: 25-28, 2008.

61. Matsumura Y, Kita S, Tanida Y, Taguchi Y, Morimoto S, Akimoto $\mathrm{K}$ and Tanaka T: Antihypertensive effect of sesamin. III. Protection against development and maintenance of hypertension in stroke-prone spontaneously hypertensive rats. Biol Pharm Bull 21: 469-473, 1998.

62. Tsuruoka N, Kidokoro A, Matsumoto I, Abe K and Kiso Y: Modulating effect of sesamin, a functional lignan in sesame seeds, on the transcription levels of lipid- and alcohol-metabolizing enzymes in rat liver: A DNA microarray study. Biosci Biotechnol Biochem 69: 179-188, 2005. 